\title{
INTEGRATED DATA PROCESSING METHODOLOGY FOR AIRBORNE REPEAT- PASS DIFFERENTIAL SAR INTERFEROMETRY
}

\author{
Changyong Dou ${ }^{\mathrm{a}, \mathrm{b}, \mathrm{c}}$, Huadong Guo ${ }^{\mathrm{c}, *}$, Chunming Han ${ }^{\mathrm{c},}$, Xijuan Yue $^{\mathrm{c}}$ and Yinghui Zhao ${ }^{\mathrm{c}}$ \\ ${ }^{\text {a }}$ Institute of Electronics, Chinese Academy of Sciences, Beijing 100190, China \\ ${ }^{\mathrm{b}}$ University of Chinese Academy of Sciences, Beijing 100049, China \\ ${ }^{a}$ Institute of Remote Sensing and Digital Earth, Chinese Academy of Sciences, Beijing 100094, China
}

\section{Commission I, WG I/1}

KEY WORDS: Airborne InSAR, residual error, time-varying baseline, ground deformation

\begin{abstract}
:
Short temporal baseline and multiple ground deformation information can be derived from the airborne differential synthetic aperture radar Interforemetry (D-InSAR). However, affected by the turbulence of the air, the aircraft would deviate from the designed flight path with high frequent vibrations and changes both in the flight trajectory and attitude. Restricted by the accuracy of the position and orientation system (POS), these high frequent deviations can not be accurately reported, which would pose great challenges in motion compensation and interferometric process. Thus, these challenges constrain its wider applications. The objective of this paper is to investigate the accurate estimation and compensation of the residual motion errors in the airborne SAR imagery and time-varying baseline errors between the diffirent data acquirations, furthermore, to explore the integration data processing theory for the airborne D-InSAR system, and thus help to accomplish the correct derivation of the ground deformation by using the airborne D-InSAR measurements.
\end{abstract}

\section{INTODUCTION}

As a major source of remote sensing data and with its unique merits of penetrating clouds and rain, and independence of sun illumination, synthetic aperture radar (SAR) has become increasingly important in Earth observation applications. Among them, Ground deformation detection is one of the important applications for the SAR interferometry (InSAR) (Massonnet and Feigl 1998). Compared with spaceborne sensors, airborne InSAR, owing to the peculiarities of its platform, features higher spatial resolution and greater flexibility in the investigation, thus, allowing it to obtain wide temporal baseline (hours to years) and multiple directions ground deformation information (Prats et al. 2008). Nevertheless, affected by the turbulences of the air, the aircraft would deviate from the designed flight path with high frequent vibrations, as well as changes in the flight trajectory, attitude and forward velocity variations. Restricted by the accuracy of the position and orientation system (POS), these high frequent diviations can not be accurately reported, which would pose great challenges in following motion compensation and interferometric process.

Uncompensated motion errors would cause artefacts in the images, among which the most important are geometric distortions and phase errors. Although, such small errors are can be neglected in most applications, this is not the case for repeatpass differential SAR interferometry (D-InSAR) applications (Zhong et al. 2012; Zhong et al. 2014). Unlike with the singlepass airborne InSAR systems, residual motion errors of each flight track are independent and do not cancel out during interferogram generation, the residual motion errors would pose great challenges to derive accurate phase information from the co-registered imagery. On the other hand, the baseline also changes with the variation of aircraft trojectories between different acquisitions, which also compounds the interferometric processing of D-InSAR system. Under current situations, the key problems of the airborne D-InSAR applications are how to accurately estimate residual motion and time-varying baseline errors from the data source, and properly compensate them in the data processing.

The objective of this paper is to investigate the integrated data processing theory for airborne D-InSAR system to correctly estimate and compensate the residual motion and time-varying errors, thus to accomplish the correct derivation of the ground deformation from airborne D-InSAR measurements.

\section{OVERVIEW THE METHODS FOR RSIDUAL MOTION ERRORS AND TIME VARYING BASELINE ESTIMATIONS}

Caused by the instablility of the aircraft, there are will be four different errors being introduced to the data processing chain which are distinguished as: residual motion errors, time-varying baseline, co-registration errors and terrain induced phase errors (Scheiber and Robert 2001). Among them, residual motion errors and time-varying baseline are the most inportant and not easily to be estimated and compensated in repeat-pass InSAR data processing.

The residual motion errors presented in the individual SAR imagery would casuse phase error and azimuth unfocusing. Furthermore, it would also impose great challenges in the correctly generating the interferogram and derive the ground deformation information. Actually, the residual motion errors are the leftover effect of the motion compensation in the SAR imaging process (Fornaro, Franceschetti, and Perna 2005). Even applying the topography- and aperture-dependent motion compensation procedure (de Macedo and Scheiber 2005), the residual errors also introduce the artifact in the the interferomgram for the repeat-pass InSAR applications. Even it can be neglected for the traditional applications, i.e., the target and change detection, and single-pass dual-antennas InSAR, for the residual errors can be canceled out in the interforemetric processing. There are typically two methods to estimate residual motion errors: one is to estimate it from an individual SAR image and the other is to estimate the error from the interferogram generated from the coregistration image pairs.

\footnotetext{
* Corresponding author: Prof. Huadong Guo. Email: hdguo@radi.ac.cn; doucy@radi.ac.cn
} 
For the first method, its objective is similar with in the motin compensation processing, namely, both of them are intent to remove of the motion errors introduced by the aircraft motion irregularities from the SAR imagery. The residual motion errors estimation methods from individual imagery can also help to improve the focusing quality in the azimuth, when the estimated residual errors are applying back to the imaging processing. Many studies have been reported on the airborne SAR motion compensation (Fornaro 1999; Fornaro, Franceschetti, and Perna 2005; Li et al. 2013; Prats, Reigber, and Mallorqui 2005; Li et al. 2012). Howerver, few literatures just focuse on the residual motion errors estimation from the individual SAR imagery. Macedo et al. (2008) proposed a autofocus algorithm based on the weighted least squares phase estimation and phase curvature autofocus extended to the range-dependent method (de Macedo, Scheiber, and Moreira 2008). This method can work well for the most of the cases, but it maybe fail when the residual motion errors is linear, which usually happens for the X- and Cband system due to the relative smaller synthetic apertures. Zhang et al. (2012, 2014) reported multsquint technique with point target (MTPT) method (Zhong et al. 2012; Zhong et al. 2014). The MTPT method is much more stable, but its applications restricted by the quality and distribution of the point targets in the scene.

For the latter method, the residual motion errors are estimated from the interferometric pair. The principle for this method is that the residual error differ in different subaperture, so it can be derive from the coregistation offset between the master and slave images by the multisequint (MS) technique (Prats and Mallorqui 2003; Scheiber and Moreira 2000; Andres and Scheiber 2006). Buulock et al. proposed a method which is only applied in the dual-antenna configuration, whose idea is also useful for repeat-pass applications (Bullock et al. 1997). Scheiber et al. estimate the azimuth coregistration offsets between two subapertures of an interferometric pair (Reigber and Papathanassiou 2001), and then through proper scaling and integration, the residual error difference of the pair can be derived from the coregistration offset (Scheiber and Moreira 2000). Based on the fact that changes in target locations simultaneously cause phase variations, the residual motion errors difference of an interferometric pair can be calculated from the phase difference between two subapertures (Prats and Mallorqui 2003; Reigber, Prats, and Mallorqui 2006). The residual motion errors estimated from phase differences are more accurate than that estimated from the coregistration offset(Bamler and Eineder 2005; Reigber, Prats, and Mallorqui 2006). For this type of approach, it's assumed that any azimuth coregistration error are from the residual motion errors. But this assumption will not be valid when a ground deformation or an along track displacement takes place in the observed scene during the two acquisitions. Aiming to overcome this problem, Prats et al. proposed an extended method to sepereate these different contributions and remove the effect of ground deformatin (Prats et al. 2009). On the other hand, the accuracy of the residual motion errors estimated by latter method is greatly impacted by the decorrelation of the observed scene (Zhong et al. 2012).

\section{METHODOLOGY}

Among the parameters for the repeat-pass airborne InSAR systems, residual motion errors and the time-varying baseline are very important and difficult to be determined, for they are caused by the aircraft deviations which can not accurately recorded by the POS system. So they have to be estimated from the data, namely, the individual SAR image and interferogram generated from the coregistrated image pair. How to accurately estimated the two parameters and compensate them correctly become the key problem for the repeat-pass airborne InSAR applications.

Commonly, the two parameters are estimated individually. For they are all resulted from the aircraft vibration and will visible as artifacts in the devived phase map, they should be be considered simutaneously in the data processing. In this paper, we proposed an integrated data processing method to estimate both the residual motion and the time-varying baseline errors. For the lack of the real experiment data source and the cost of the flight campaign is very high, the semi-physical simulation data (Dou et al. 2014) will be used to conduct the study.

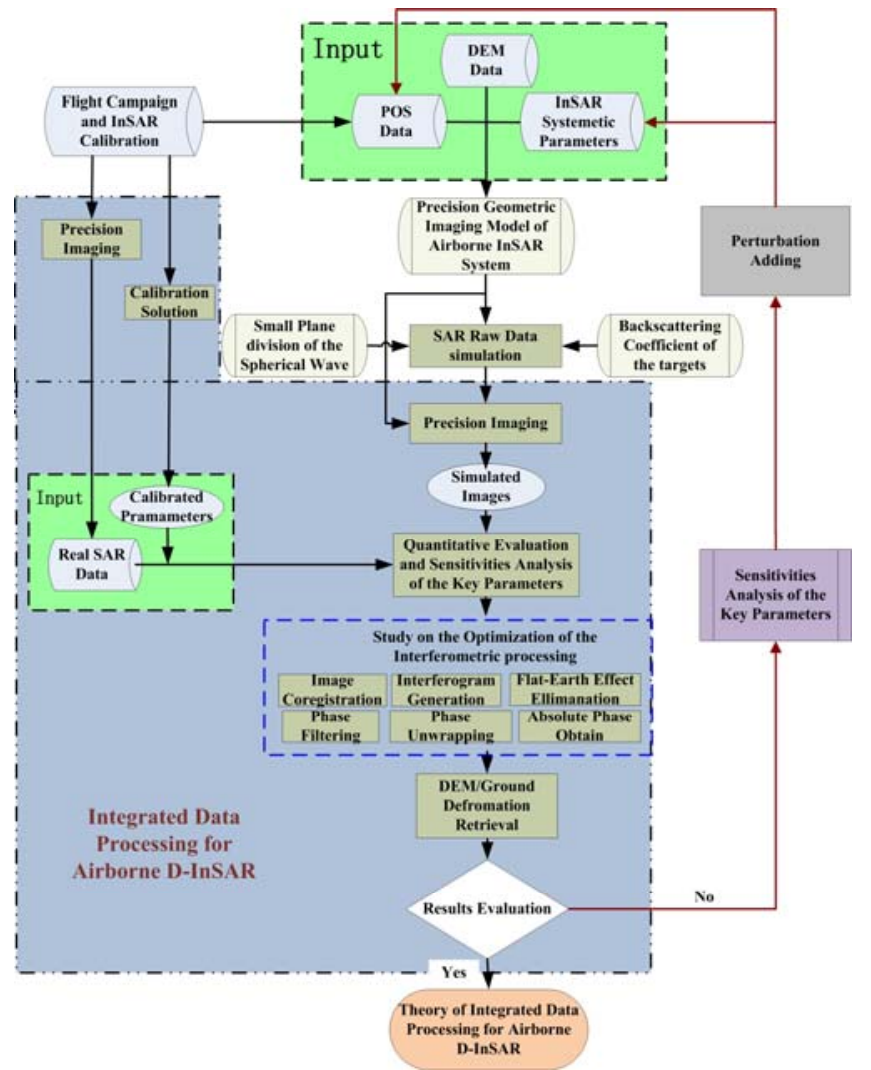

Fig. 1. Overall research scheme of airborne D-InSAR data integrated processing based on semi-physical simulation and real data

The overall research scheme of airborne D-InSAR data integrated processing is shown in Fig. 1. The input data of the research scheme are from a real flight campaign using the newly developed dual-antinnas InSAR system. A fine resolution ( 0.5 meter) of the digital elevation model (DEM) with accuracies of $0.2,1.2$ and 0.5 meter in the range, azimuth and elevation directions, respectively, were generated from the InSAR dataset acquired in the flight compaign (Guo et al.). The DEM, along with the InSAR parameters and POS data, was used to generate the simulated SAR raw signal data based on regious geometric imaging model of airborne InSAR system. Based on the geometric imaging model, the installation offset between the Global Positioning System (GPS), Inertial Measurement Unit (IMU) and the InSAR antennas are taken care of, thus, the precise geometric relationship of the airborne D-InSAR system can be guaranteed (Dou et al. 2014). The simulated SAR raw signal data was used to generate the simulated SAR images by the precision imaging process. Combining the simulated SAR imagey and precisely calibrated 
real imagery (Han et al. 2014) acquired in the flight campaign, quantitative evaluation and sensitivites analysis of the key parameters of the airborne SAR systme were performed. In order to investigate data processing theory for the airborne DInSAR application, the perturbations of the aircraft trajectory and attitude are gradually added to the input data, thus a closed feed back loop is completed. Among the investigations, the key part is how to accurately estimate the residual motion error and time-varying baseline, and compensate them efficiently.

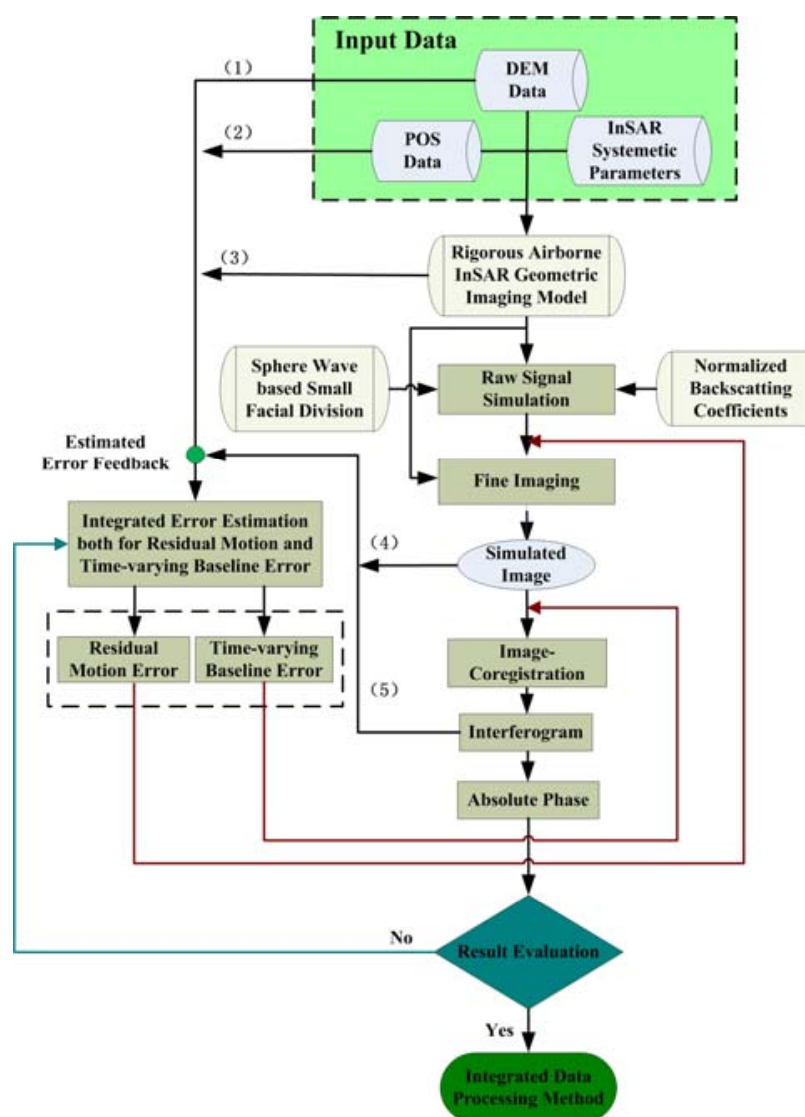

Fig. 2. Scheme of integrated estimation of residual motion and time-varying baseline errors.

As shown in Fig. 2, the integrated estimation of both residual motion and the time-varying baseline errors are performed by using the simulated imagey, interferogram, DEM, POS and rigorous geometric imaging model of airborne InSAR system. Different from the traditional method, the residual motion and the time-varying baseline errors are simutineously estimated from the above mentioned image and interferogram under the help of DEM, POS and the rigorous geometric imaging model. This idea is one of two noveltis of the our method, using five data source to study the two objectives. Because the unrecorded aircraft deviations are contained in the SAR image and interferogram generated from the coregistrated images, the residual motion and time-varying baseline errors have to be estimated from these data, which is named as data based retrival method. By this approach, it's impossible to accuratelly determine these errors at one time. Therefor, an iterative estimation method is essential to accurately retrive these errors. An iterative closed loop is used in our study, as shown in Fig. 2. The estimated residual error will fed back to the "fine imaging" step in the data processing chain to compenste the errors in the imagey. At the same time, the time-varying baseline will fed back to the "interferogram generation" step to improve the interferogram quality. Then, the newly imagery and interferogram will used to estimate the residual motion and time-varying baseline errors again. This iterative loop will stop until the error falls into a certain threshhold. The concept of the iterative colsed loop is the sencond novelty of our method. These two novelties, along with closed loop overall research strategy, reflect the concept of the "integrated" procesing for airborne D-InSAR system.

\section{CONCLUSION}

An integrated data processing method for the airborne D-InSAR applications are proposed in this paper. Firstely, the overview of the studies in this field is introduced. Then, the overall research strategy is addressed, whos input data is based on the semi-physical simulation data and real data acquired in the flight campaign. The semi-physical simulation can provide controllable and good quality data source for the study. To ensure the precise geometric relationship of the airborne DInSAR system, regious geometric imaging model are set up to take care of the installation offset position between the GPS antenna, IMU and the InSAR antennas in the aircraft bodyfixed coordinate system. Aiming to accurately estimate the residual motion and time-varying baseline errors and compensate them, an integrated estimation method is proposed. There are two noveltis contained in this approach: firstly, using five data source to study the two objectives, namely, using the SAR imagery, interferogram, DEM, POS data and regorous geometric imaging model to study resicual motion and timevarying baseline errors; secondly, iterative closed loop to gradually compensate the errors. After this integrated data processing methodogy are trained and validated by the controllable simulated data, the integrated data processing theory for the airborne D-InSAR syetem can be estiblished and can be applied to the real applications.

\section{ACKNOWLEDGEMENTS}

This work was supported in part by the Director Youth Foundation of Institute of Remote Sensing and Digital Earth, Chinese Academy of Sciences (Y3SJ6000CX), the National Natural Science Foundation of China under contract 61132006 and 41201481, and fundamental research project of Chinese academy of sciences (KZZD-EW-TZ-18).

\section{REFERENCES}

Andres, C., and R. Scheiber. 2006. Inversion of residual motion errors in airborne single and repeat pass interferometry in the presence of squint and large topography variations. In EUSAR'06.

Bamler, R., and M. Eineder. 2005. Accuracy of differential shift estimation by correlation and split-bandwidth interferometry for wideband and delta-k SAR systems. IEEE Transactions on Geoscience and Remote Sensing 2 (2), pp.151-155.

Bullock, R., R. Voles, A. Currie, H. Griffiths, and P. Brennan. 1997. Two-look method for roll correction of aircraft-borne interferometric SAR data. In IEE Colloq. Radar Interferometry.

de Macedo, K. A. C., and Rolf Scheiber. 2005. Precise topography- and aperture-dependent motion compensation for airborne SAR. IEEE Transactions on Geoscience and Remote Sensing, 2 (2), pp.172 - 176. 
de Macedo, K.A.C., R. Scheiber, and A. Moreira. 2008. An Autofocus Approach for Residual Motion Errors With Application to Airborne Repeat-Pass SAR Interferometry. IEEE Transactions on Geoscience and Remote Sensing, 46 (10), pp.3151 - 3162.

Dou, Changyong, Huadong Guo, Chunming Han, yuquan1 Liu, Xijuan Yue, and Yinghui Zhao. 2014. Semi-physical Simulation of the Airborne InSAR based on Rigorous Geometric Model and Real Navigation Data. Paper read at IOP Conf. Ser.: Earth Environ. Sci. 35th International Symposium on Remote Sensing of Environment (ISRSE35).

Fornaro, G. 1999. Trajectory deviations in airborne SAR: analysis and compensation. IEEE Transactions on Aerospace and Electronic Systems, 35 (3), pp.997 - 1009.

Fornaro, G., G. Franceschetti, and S. Perna. 2005. Motion compensation errors: effects on the accuracy of airborne SAR images. IEEE Transactions on Aerospace and Electronic Systems, 41 (4), pp.1338 - 1352

Guo, Huadong, Chunming Han, Xijuan Yue, and Changyong Dou. Production of a wide-area DSM and DOM using the newly developed high-resolution airborne InSAR system. Submitted to IEEE Transactions on Geoscience and Remote Sensing.

Han, Chunming, huadong Guo, Xijuan Yue, Changyong Dou, Mingming Song, and Yanbing Zhang. 2014. Calibration and Validation of Airborne InSAR Geometric Model. Paper read at IOP Conf. Ser.: Earth Environ. Sci. 35th International Symposium on Remote Sensing of Environment (ISRSE35).

Li, Yake, Chang Liu, Yanfei Wang, and Qi Wang. 2012. A Robust Motion Error Estimation Method Based on Raw Data. IEEE Transactions on Geoscience and Remote Sensing, 50 (7), pp. $2780-2790$.

Li, Yan-lei, Xing-dong Liang, Chi-biao Ding, Liang-jiang Zhou, and Quan Ding. 2013. Improvements to the Frequency Division-Based Subaperture Algorithm for Motion Compensation in Wide-Beam SAR. IEEE Geoscience and Remote Sensing Letters, 10 (5), pp. 1219 - 1223.

Massonnet, Didier, and Kurt L. Feigl. 1998. Radar Interferometry and its application to changes in the Earth's Surface. Reviews of Geophysics, 36, pp. 441-500.

Prats, P. , and J.J. Mallorqui. 2003. Estimation of azimuth phase undulations with multisquint processing in airborne interferometric SAR images. IEEE Transactions on Geoscience and Remote Sensing, 41 (6), pp. 1530 - 1533.

Prats, P., R. Scheiber, A. Reigber, C. Andres, and R. Horn. 2009. Estimation of the Surface Velocity Field of the Aletsch Glacier Using Multibaseline Airborne SAR Interferometry. IEEE Transactions on Geoscience and Remote Sensing, 47 (2), pp. 419 - 430.

Prats, P., A. Reigber, and J.J. Mallorqui. 2005. Topographydependent motion compensation for repeat-pass interferometric SAR systems. IEEE Geoscience and Remote Sensing Letters, 2 (2), pp. 206 - 210.
Prats, P., A. Reigber, J.J. Mallorqui, R. Scheiber, and A. Moreira. 2008. Estimation of the Temporal Evolution of the Deformation Using Airborne Differential SAR Interferometry. IEEE Transactions on Geoscience and Remote Sensing, 46 (4), pp. 1065 - 1078.

Reigber, A., and K. Papathanassiou. 2001. Correction of residual motion errors in airborne repeat-pass interferometry. In IGARSS'01.

Reigber, A., P. Prats, and J.J. Mallorqui. 2006. Refined estimation of time-varying baseline errors in airborne SAR interferometry. IEEE Geoscience and Remote Sensing Letters, 3 (1), pp. 145-149.

Scheiber, R. , and P. Robert. 2001. Origin and correction of phase errors in airborne repeat-pass SAR interferometry In IGARSS'01.

Scheiber, R., and A. Moreira. 2000. Coregistration of interferometric SAR images using spectral diversity. IEEE Transactions on Geoscience and Remote Sensing, 38 (5), pp. 2179 - 2191.

Zhong, Xuelian, Huadong Guo, Maosheng Xiang, and Huanyin Yue. 2012. Residual motion estimation with point targets and its application to airborne repeat-pass SAR interferometry. International Journal of Remote Sensing, 33 (3), pp. 762-780.

Zhong, Xuelian, Maosheng Xiang, Huanyin Yue, and Huadong Guo. 2014. Algorithm on the Estimation of Residual Motion Errors in Airborne SAR Images. IEEE Transactions on Geoscience and Remote Sensing, 52 (2), pp. 1311 - 1323. 